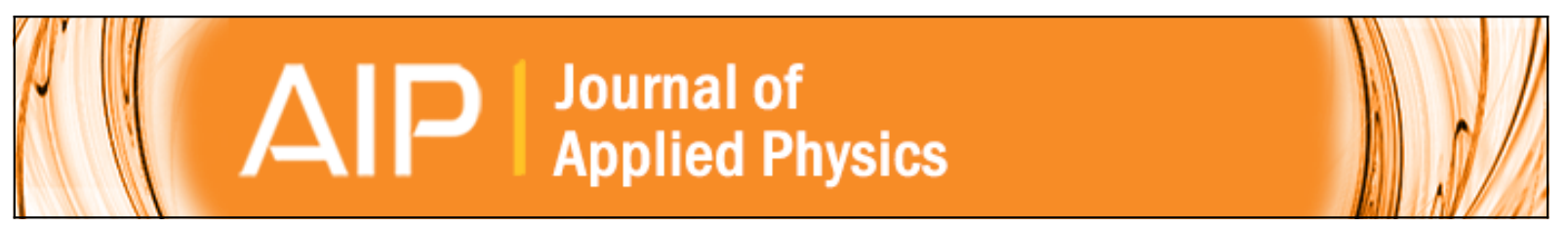

\title{
The quest for inorganic fullerenes
}

Susanne Pietsch, Andreas Dollinger, Christoph H. Strobel, Eun Ji Park, Gerd Ganteför, Hyun Ook Seo, Young

Dok Kim, Juan-Carlos Idrobo, and Stephen J. Pennycook

Citation: Journal of Applied Physics 118, 134302 (2015); doi: 10.1063/1.4932143

View online: http://dx.doi.org/10.1063/1.4932143

View Table of Contents: http://scitation.aip.org/content/aip/journal/jap/118/13?ver=pdfcov

Published by the AIP Publishing

\section{Articles you may be interested in}

Controlled growth of vertically aligned $\mathrm{MoO} 3$ nanoflakes by plasma assisted paste sublimation process

J. Appl. Phys. 114, 184310 (2013); 10.1063/1.4830278

Direct transfer of gold nanoislands from a MoS 2 stamp to a Si-H surface

J. Vac. Sci. Technol. B 28, 484 (2010); 10.1116/1.3385674

Fabrication and optical properties of Ti-doped W18049 nanorods using a modified plasma-arc gas-condensation techniquea)

J. Vac. Sci. Technol. B 27, 2170 (2009); 10.1116/1.3208007

Synthesis and Properties of Alkali Metal Intercalated Fullerene-like MS2 (M=W,Mo) Nanoparticles

AIP Conf. Proc. 633, 67 (2002); 10.1063/1.1514076

Structure and electronic properties of carbon onions

J. Chem. Phys. 114, 7477 (2001); 10.1063/1.1360197

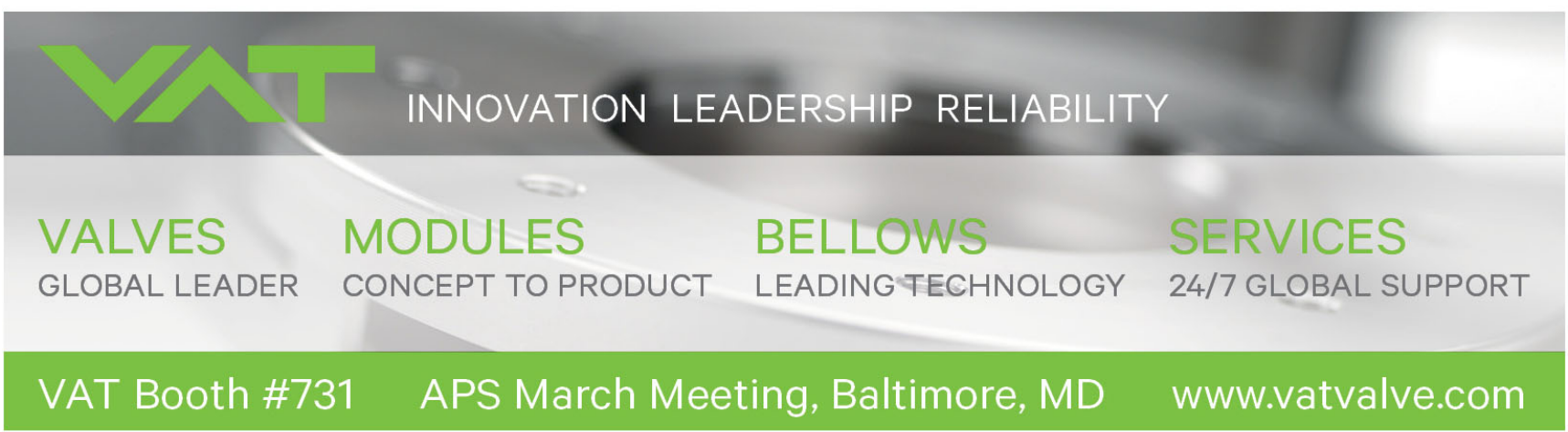




\title{
The quest for inorganic fullerenes
}

\author{
Susanne Pietsch, ${ }^{1}$ Andreas Dollinger, ${ }^{1}$ Christoph H. Strobel, ${ }^{1}$ Eun Ji Park, ${ }^{2}$ \\ Gerd Ganteför, ${ }^{1, a)}$ Hyun Ook Seo, ${ }^{3}$ Young Dok Kim, ${ }^{2, a)}$ Juan-Carlos Idrobo, ${ }^{4}$ \\ and Stephen J. Pennycook ${ }^{5}$ \\ ${ }^{1}$ Department of Physics, University of Konstanz, D-78457 Konstanz, Germany \\ ${ }^{2}$ Department of Chemistry, Sungkyunkwan University, 440-746 Suwon, South Korea \\ ${ }^{3}$ Center for Free-Electron Laser Science/DESY, D-22607 Hamburg, Germany \\ ${ }^{4}$ Center for Nanophase Materials Sciences, Oak Ridge National Laboratory, Oak Ridge, \\ Tennessee 37831, USA \\ ${ }^{5}$ Department of Materials Science and Engineering, National University of Singapore, Singapore 117575
}

(Received 20 August 2015; accepted 20 September 2015; published online 2 October 2015)

Experimental results of the search for inorganic fullerenes are presented. $\mathrm{Mo}_{\mathrm{n}} \mathrm{S}_{\mathrm{m}}{ }^{-}$and $\mathrm{W}_{\mathrm{n}} \mathrm{S}_{\mathrm{m}}{ }^{-}$ clusters are generated with a pulsed arc cluster ion source equipped with an annealing stage. This is known to enhance fullerene formation in the case of carbon. Analogous to carbon, the mass spectra of the metal chalcogenide clusters produced in this way exhibit a bimodal structure. The species in the first maximum at low mass are known to be platelets. Here, the structure of the species in the second maximum is studied by anion photoelectron spectroscopy, scanning transmission electron microscopy, and scanning tunneling microcopy. All experimental results indicate a two-dimensional structure of these species and disagree with a three-dimensional fullerene-like geometry. A possible explanation for this preference of two-dimensional structures is the ability of a two-element material to saturate the dangling bonds at the edges of a platelet by excess atoms of one element. A platelet consisting of a single element only cannot do this. Accordingly, graphite and boron might be the only materials forming nano-spheres because they are the only single element materials assuming two-dimensional structures. @ 2015 AIP Publishing LLC.

[http://dx.doi.org/10.1063/1.4932143]

\section{INTRODUCTION}

Nanoparticles and clusters have unique physical and chemical properties that make them interesting for basic research as well as applications. Perhaps, the best known clusters are the carbon fullerenes. ${ }^{1}$ These are hollow spheres of pure carbon, which are available in various sizes. The driving force for the formation of such spheres is the energetically unfavorable position of carbon atoms at the edges of graphene planes. For a graphene flake, it is therefore advantageous to curve itself into a sphere, so that there is no edge. The most stable fullerenes are $\mathrm{C}_{60}$ and $\mathrm{C}_{70}$, but there is a variety of other sizes, from which a manifold of carbon-derived materials can be synthesized. Additional atoms or clusters can be stored in the hollow spheres (endohedral), stabilizing the fullerenes even further. ${ }^{2}$ The question might be raised whether there are other materials forming hollow nanospheres. In principle, all two-dimensional materials ${ }^{3}$ consisting of weakly interacting monomolecular planes could behave similarly. A possible candidate is boron. Within a certain size range, elemental boron clusters assume a twodimensional structure. Recently, this approach led to the discovery of the all-boron fullerenes $\mathrm{B}_{40}$ and $\mathrm{B}_{39}{ }^{4,5}$ In addition to the single-element materials carbon and boron, there are many other materials that consist of two or more elements and have a two-dimensional structure. However, until today, no other so-called inorganic fullerenes have been found.

\footnotetext{
a) Authors to whom correspondence should be addressed. Electronic addresses: gerd.gantefoer@uni-konstanz.de and ydkim91@skku.edu
}

There are many materials that form nanotubes and nested fullerenes similar to carbon, ${ }^{6-17}$ but so far, no "single-wall" nano-spheres analogous to $\mathrm{C}_{60}$ and $\mathrm{B}_{40}$ have been observed.

$\mathrm{MoS}_{2}$ and $\mathrm{WS}_{2}$ are layered materials which exhibit a certain similarity to carbon. ${ }^{3}$ Both are used as lubricants, because the weak van-der-Waals interaction between the layers makes these materials soft and friction reducing. Both materials easily form nanotubes again similar to carbon. ${ }^{18}$ Also, both materials form nested fullerenes, ${ }^{10,19}$ which can be viewed as "multiwall" fullerenes. The smallest such structure seems to have three walls. ${ }^{10,20}$ In contrast to carbon, these structures are not spherical. The smallest ones have octahedral geometries. ${ }^{17}$ An octahedron is built from eight planar triangles. Triangular two-dimensional nano-platelets of $\mathrm{MoS}_{2}$ and $\mathrm{WS}_{2}$ are exceptional stable. ${ }^{21,22}$ There are magic numbers, i.e., triangles with a certain size that are more stable than others. In the gas phase, the most stable platelet is $\mathrm{W}_{15} \mathrm{~S}_{42}$ and the smallest observed platelet is $\mathrm{W}_{10} \mathrm{~S}_{30}{ }^{23-25}$ The octahedral shape of the nested inorganic fullerenes indicates that two-dimensional triangular structures are preferred by $\mathrm{MoS}_{2}$ and $\mathrm{WS}_{2}$. No "single-wall" real fullerenes have been found until today.

Carbon fullerenes have first been discovered in a mass spectrum of clusters generated by a cluster ion source. ${ }^{26,27}$ The material (carbon) is vaporized in a laser pulse and cooled in an inert carrier gas. Condensation results in the formation of dimers and trimers and later larger clusters. A few percent of the clusters is charged allowing the recording of mass spectra. The fullerene mass spectrum exhibits a 
characteristic bimodal structure. The first maximum at low mass corresponds to the carbon chains and rings, while the second maximum is formed by the fullerenes. Within this second maximum, the mass with $720 \mathrm{amu}\left(=\mathrm{C}_{60}\right)$ stands out. So far, cluster methods have not been used for a systematic search for fullerenes made from $\mathrm{MoS}_{2}$ or $\mathrm{WS}_{2}$. Nanoparticles have been synthesized by other techniques suitable for the synthesis of large amounts of material. One common method is the reduction of a metal oxide $\left(\mathrm{MoO}_{3}\right.$ or $\left.\mathrm{WO}_{3}\right)$ in an $\mathrm{H}_{2} / \mathrm{H}_{2} \mathrm{~S}$ atmosphere. ${ }^{28}$ The synthesized particles consist of thousands of atoms and are much larger than carbon fullerenes. With these methods, it is difficult to control the condensation process and optimize for the generation of clusters less than 100 atoms. A few attempts have been made to synthesize smaller particles and record mass spectra. ${ }^{29}$ However, the results of these earlier experiments do not agree with the data presented here.

We present the results of a search for inorganic fullerenes by using well established methods of cluster science. Nanoclusters of $\mathrm{Mo}_{n} \mathrm{~S}_{\mathrm{m}}$ and $\mathrm{W}_{\mathrm{n}} \mathrm{S}_{\mathrm{m}}$ are synthesized in a cluster ion source at conditions under which in the case of graphite fullerenes are formed. ${ }^{30,31}$ The mass spectra show a bimodal distribution similar to the mass spectra of the carbon fullerenes. It is already known that the particles in the first maximum are one-dimensional chains and two-dimensional platelets. $^{23-25}$ Here, we focus on the structure determination of the larger clusters in the second maximum. These clusters are studied with anion photoelectron spectroscopy, scanning transmission electron microscopy, and scanning tunneling microscopy. All experimental data support the theoretical prediction that these particles do not have a fullerene-like 3D-structure but are two-dimensional platelets. At the end of the paper, it will be discussed why carbon and boron fullerenes may be the only fullerenes existing.

\section{EXPERIMENTAL}

Neutral and negatively charged $\mathrm{Mo}_{\mathrm{n}} \mathrm{S}_{\mathrm{m}}$ and $\mathrm{W}_{\mathrm{n}} \mathrm{S}_{\mathrm{m}}$ clusters are generated with a pulsed arc cluster ions source (PACIS). ${ }^{23-25}$ In the past, the source has been used successfully to synthesize all kinds of carbon fullerenes. ${ }^{30,31}$ In contrast to the generation of metal clusters, for the effective generation of carbon fullerenes, an annealing stage is necessary. ${ }^{30,32}$ It is mounted on the end of the extender of the source (Fig. 1). The annealing stage is a weak electrical discharge which reheats the carrier gas containing the carbon clusters. In the primary strong electrical discharge, huge amounts of carbon are vaporized which condense downstream from the extender. This results in the formation of large graphitic carbon clusters. The annealing heats this ensemble of large clusters up again. Above the mass of $\mathrm{C}_{30}$, which is the minimum size of stable fullerenes under these conditions, fullerenes are practically the only species generated. Without the annealing, only very few fullerenes are formed. This technique is now applied to generate inorganic fullerenes.

Metalsulfide clusters are generated by the vaporization of the corresponding metal ( $\mathrm{W}$ or Mo), while a carefully controlled amount of $\mathrm{H}_{2} \mathrm{~S}$ is introduced into the PACIS. This method has been already used successfully to synthesize $\mathrm{Mo}_{\mathrm{n}} \mathrm{S}_{\mathrm{m}}$ and $\mathrm{W}_{\mathrm{n}} \mathrm{S}_{\mathrm{m}}$ platelets and nanowires. ${ }^{23-25}$ No indication for hydrogen contamination has been found in the mass spectra or in the photoelectron spectra. $\mathrm{H}_{2} \mathrm{~S}$ based methods have been used by other groups searching for inorganic fullerenes and no indications for remaining hydrogen have been found. ${ }^{12,26}$ The metal-sulfur interaction is so dominant that hydrogen is driven off.

The cluster anions are mass-selected by a reflectron timeof-flight mass spectrometer. A selected bunch of anions can be studied by photoelectron spectroscopy. The anions are irradiated by a laser pulse from a $\mathrm{F}_{2}$-Excimer laser with a photon energy of $7.9 \mathrm{eV}$. This relatively high photon energy is required because of the high electron affinities of the sulfides. The kinetic energy of the detached electrons is measured using a magnetic bottle time-of-flight electron spectrometer. This part of the apparatus is described elsewhere. ${ }^{33}$

For studies with a scanning transmission electron microscope (STEM), neutral clusters are deposited on a standard TEM grid consisting of a copper grid holding with a thin silicon dioxide membrane (Fig. 1). After deposition, the wafer is removed from the vacuum and sent to the STEM group at Oak Ridge National Laboratory. There, the sample is studied with a high resolution aberration corrected STEM. With this instrument, it is even possible to identify single atoms. In a different setup, mass-selected clusters are deposited on highly oriented pyrolytic graphite (HOPG). The samples are mounted at the end the reflectron time-of-flight massspectrometer. A pulsed mass gate allows controlling the size of the clusters that reach the surface. The kinetic energy of

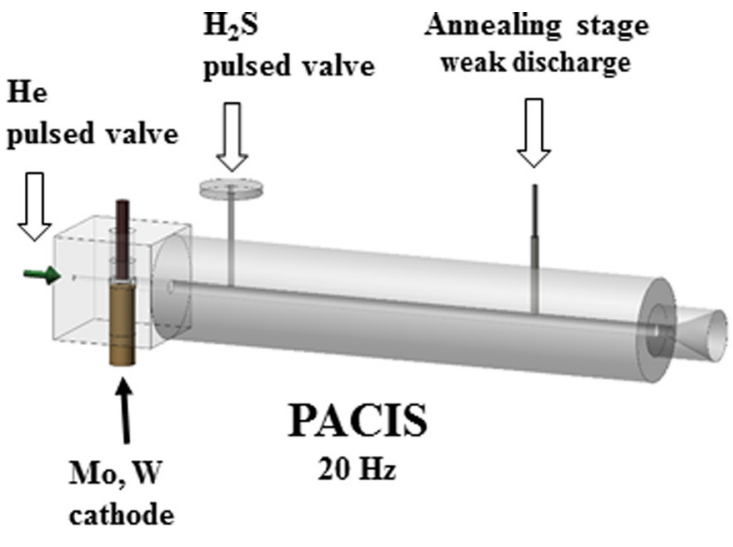

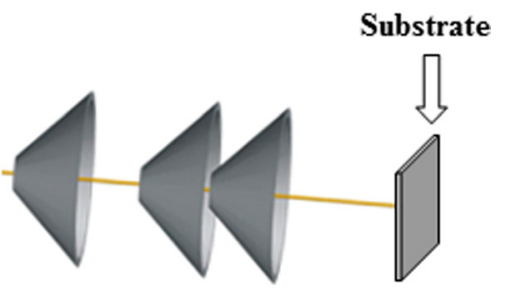

Skimmers differential pumping
FIG. 1. PACIS source with the annealing stage and the setup for neutral cluster deposition on a TEM grid. 
the cluster ions impacting or landing on the surface can be changed by adjusting the sample potential. This is not a sophisticated soft-landing cluster deposition setup, because in the time-of-flight mass spectrometer, ions have a rather broad kinetic energy distribution. Hence, even if a low kinetic energy is selected in an attempt to "soft-land" the clusters, some species still have relatively high kinetic energies and others turn around in the deceleration field before reaching the surface. At such conditions, some clusters are soft landed, while others impact with considerably excess energy onto the surface. After deposition, the samples are transferred in a small vacuum chamber $\left(10^{-7}\right.$ mbar) to an ultrahigh vacuum scanning tunneling microscope (STM).

\section{RESULTS AND DISCUSSION}

Fig. 2 displays mass spectra of annealed $\mathrm{Mo}_{\mathrm{n}} \mathrm{S}_{\mathrm{m}}$ and $\mathrm{W}_{\mathrm{n}} \mathrm{S}_{\mathrm{m}}$ cluster anions. Without the annealing, only one maximum is observed, which starts at low masses and extends up at very high masses (not shown). With the annealing, two maxima are observed. The clusters in the first maximum at low mass have previously been studied by photoelectron spectroscopy. ${ }^{23-25}$ The experimental data have been compared to corresponding calculations. These species are two-dimensional platelets. In addition, intense lines may appear at low mass (Fig. 2, upper trace). These are very small $\left(\mathrm{MoS}_{2}\right)_{\mathrm{n}}{ }^{-}$and $\left(\mathrm{WS}_{2}\right)_{\mathrm{n}}{ }^{-}$clusters with $\mathrm{n} \sim 1-10$. Their appearance is highly depending on the source conditions. The position of the second mass spectrum depends on the source conditions, too (Fig. 3). Several parameters control the average size: the intensity of the main discharge determining the amount of vaporized metal, the amount of $\mathrm{H}_{2} \mathrm{~S}$ introduced into the source, the amount of helium and the relative timing of the main discharge and the annealing discharge. In general, with increasing time, the clusters spend in the extender and with an increasing amount of

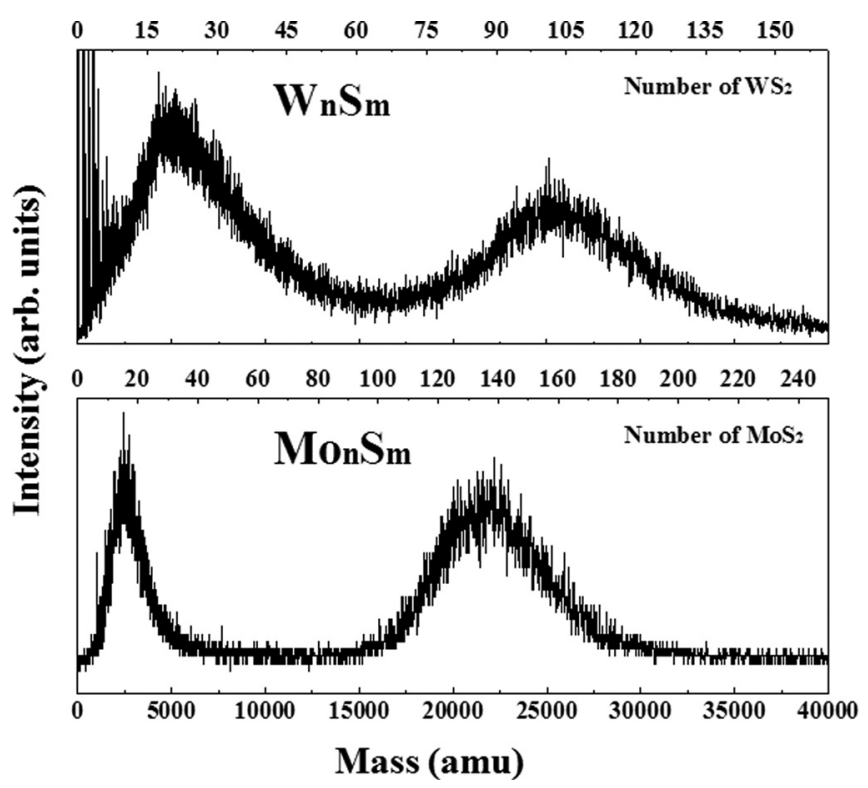

FIG. 2. Typical mass spectra of annealed $\mathrm{W}_{\mathrm{n}} \mathrm{S}_{\mathrm{m}}{ }^{-}$and $\mathrm{Mo}_{\mathrm{n}} \mathrm{S}_{\mathrm{m}}{ }^{-}$cluster anions generated with the PACIS equipped with the annealing stage. With the annealing switched off, just one broad maximum is observed (not shown).

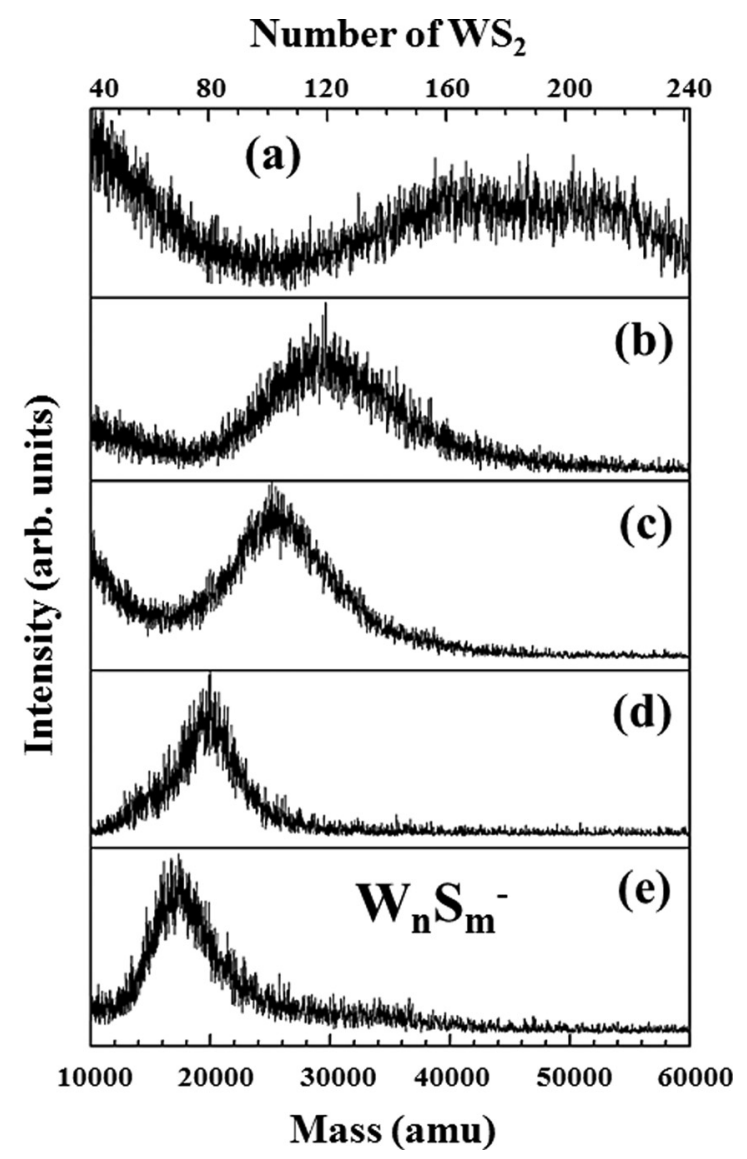

FIG. 3. Mass spectra of $\mathrm{W}_{\mathrm{n}} \mathrm{S}_{\mathrm{m}}{ }^{-}$cluster anions recorded at different source conditions. The most important parameters are the time the cluster anions spend in the extender ((a) and (b): $1000 \mu \mathrm{s}$; (c) and (d): $600 \mu \mathrm{s}$; (e): $450 \mu \mathrm{s})$ and the amount of $\mathrm{H}_{2} \mathrm{~S}$ added to the He seeding gas ((a): $25 \%$; (b) and (c): 20\%; (d): $15 \%$; (e): $13 \%$ ). The second maximum can easily be shifted to larger masses. At lower masses, there seems to exist a minimum size corresponding to the mass of approximately 60 monomer units. If the parameters are optimized to shift the maximum to a size below $\sim 80$ monomers, it becomes weaker and disappears.

$\mathrm{H}_{2} \mathrm{~S}$, the clusters grow larger. On the other hand, there seems to be a minimum size analogous to the case of carbon fullerenes. ${ }^{30}$ If the parameters are optimized to shift the second maximum to mass lower than approximately 80 monomers, this maximum disappears. The minimum size is about $60 \mathrm{MoS}_{2}$ or $\mathrm{WS}_{2}$ monomers. However, there is no clear cut off. The maximum just loses intensity and finally disappears.

The apparent existence of a lower mass limit for the second maximum fits to the hypothesis that these species might have a three-dimensional fullerene-like structure. For carbon, the lower mass limit of the fullerenes generated with this method is $\mathrm{C}_{30}$. There are smaller fullerenes, but their abundance is extremely low. In principle, the inorganic fullerenes could be either spherical or octahedral. In the spherical particles, the monomolecular plane needs to be bent. The smaller the diameter the more energy is required. Alternatively, the particles could be octahedral as indicated by the TEM pictures of nested inorganic fullerenes. ${ }^{10}$ Such a cluster can be viewed as eight triangular platelets assembled to an octahedron. For the platelets, there is a minimum size. The smallest observed platelet consists of ten $\mathrm{MoS}_{2}$ units plus a less well defined 
number of excess sulfur atoms stabilizing the edges (e.g., $\left.\mathrm{Mo}_{10} \mathrm{~S}_{30}\right){ }^{24}$ This would give a lower limit of $80 \mathrm{MoS}_{2}$ units for a hypothetical octahedral cluster. The experiments indicate a lower mass limit of 60 units. Since the lower mass limit for the platelets is very pronounced-there are no smaller platelets in the gas phase-our observation of masses lower than $80 \mathrm{MoS}_{2}$ units contradicts the hypothesis of an octahedral three-dimensional geometry.

The mass resolution is not sufficient to resolve individual cluster sizes within the second maximum. Therefore, a series of photoelectron spectra have been recorded by scanning the mass in equidistant steps over the range covered by the second maximum. The results are displayed in Fig. 4. All photoelectron spectra look similar and are basically featureless. The small peaks visible in several spectra are noise and not reproducible. There is a single broad maximum shifting slowly towards higher binding energy with increasing mass. Despite all efforts, no hint of a "special" photoelectron spectrum could be found. With the same method, $\mathrm{C}_{60}$ or $\mathrm{B}_{40}$ would have been easily identified because of their large HOMO-LUMO gaps. In the anion photoelectron spectra, such a gap gives rise to a separate peak at low binding energy. Such a peak would be noticed in the series of measurements displayed in Fig. 4. However, no such peak has been found. It is possible that such features are too weak,

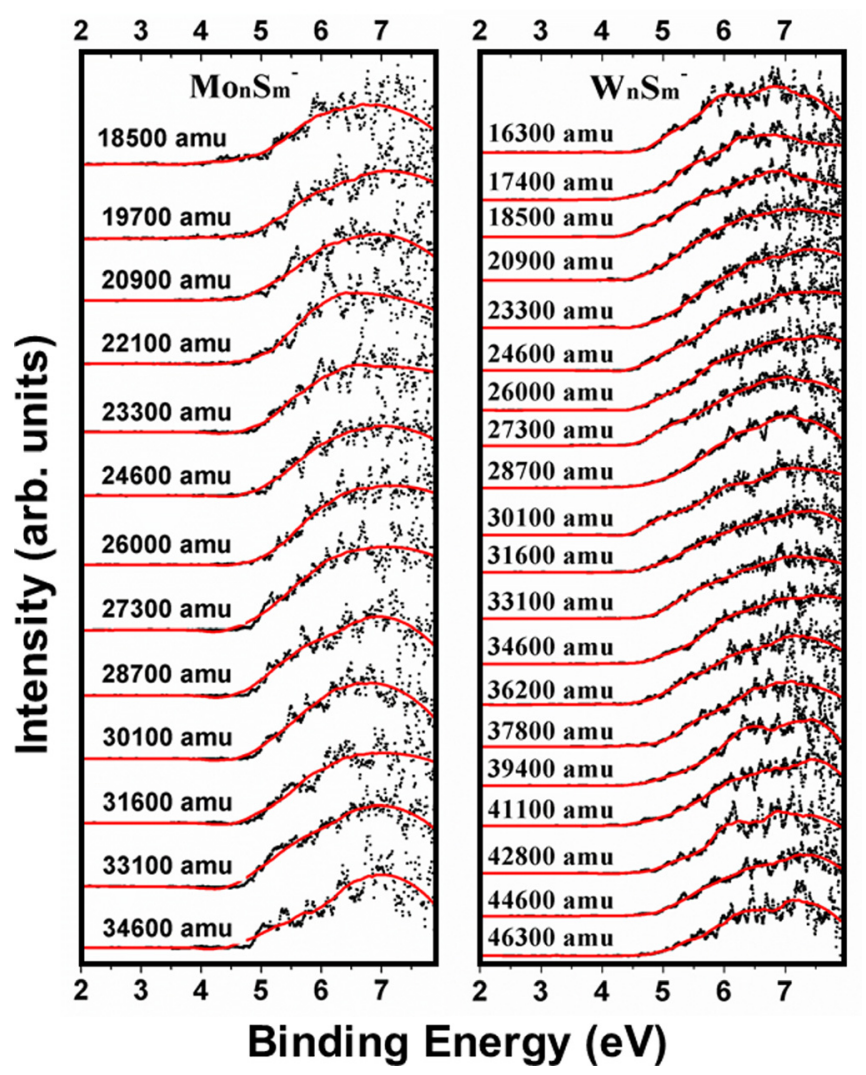

FIG. 4. Photoelectron spectra of cluster anions within the second maximum recorded with a photon energy of $7.9 \mathrm{eV}$. The mass resolution is not sufficient to resolve individual masses. Therefore, the mass range has been covered by a systematic scan. The mass is given in amu for each spectrum. The red lines are averaged spectra (averaged over $150\left(\mathrm{~W}_{\mathrm{n}} \mathrm{S}_{\mathrm{m}}{ }^{-}\right)$or 90 dots $\left(\mathrm{Mo}_{\mathrm{n}} \mathrm{S}_{\mathrm{m}}{ }^{-}\right)$). With increasing mass, the position of the broad maximum shifts to higher binding energy. especially if the corresponding cluster anion has a low relative abundance. However, a stable cluster should be one with an enhanced abundance. Therefore, we conclude that our photoelectron data do not support the assumption of the existence of any stable "magic" species within the second maximum of the mass spectrum.

Since the gas phase studies did not provide any clue on the geometric structure of the species in the second maximum, the clusters are deposited on surfaces and studied with microscopy techniques. In a first attempt, the neutral clusters exiting the PACIS in forward direction are deposited on a standard TEM grid. It consists of a $\mathrm{Cu}$ grid holding a thin membrane of silicon dioxide (Fig. 1). The source is optimized for maximum production of clusters in the second maximum. Then, the sample is exposed to the neutral beam for $10-60 \mathrm{~s}$. Fig. 5 displays three STEM pictures obtained with this method. The deposited material has agglomerated into larger lumps of material. In the two pictures recorded at lower resolution, 5(a) and 5(b), material with a layered structure is observed. Such structures are typical for bulk-like $\mathrm{WS}_{2}$. At highest resolution, 5(c), very small stacks of platelets can be discerned (marked with a white ellipse in the upper right corner). All STEM pictures show only twodimensional bulk-like structures or structureless amorphous material. Nothing resembling a three-dimensional fullerenelike structure could be found.

In a final attempt to gain more information about the geometric structure of the species in the second maximum, mass-selected cluster ions have been deposited after passage through the time-of-flight mass spectrometer. There are several difficulties involved into this approach. In the time-offlight instrument, the anions are accelerated up to $2.5 \mathrm{keV}$. For deposition, the anions need to be decelerated. However, in a time-of-flight instrument, the anions do not have a welldefined kinetic energy. Instead, they have a rather broad distribution of kinetic energies. If the anions are decelerated in an electric field before they reach the sample, some species might still have a considerable excess energy of several $100 \mathrm{eV}$ when they hit the surface. Others with low kinetic energy may turn around before reaching the surface. Another difficulty is the extremely low intensity of the deposited material. Even after running the machine for several hours, only a few clusters could be found on the surface. A pulsed mass gate allows for a mass selection. For intensity reasons, the mass range was selected to cover the full range of the second maximum in the mass spectrum (see Fig. 2). After deposition, the samples are transferred to a scanning tunneling microscope operated in ultrahigh vacuum, without being exposed to air.

Fig. 6 displays a STM image of cluster anions deposited without deceleration. The kinetic energies are between 3 and $7 \mathrm{eV} /$ atom. The clusters are pinned to surface defects created by the impact. The STM is not able to resolve fine structures within such small particles. However, it is possible to determine the height of particles on flat surfaces with considerable accuracy. The height scans for three particles visible in Fig. 6 are displayed, too. The heights correspond more or less to the height of a single layer of $\mathrm{MoS}_{2}(\sim 0.5 \mathrm{~nm})$. 

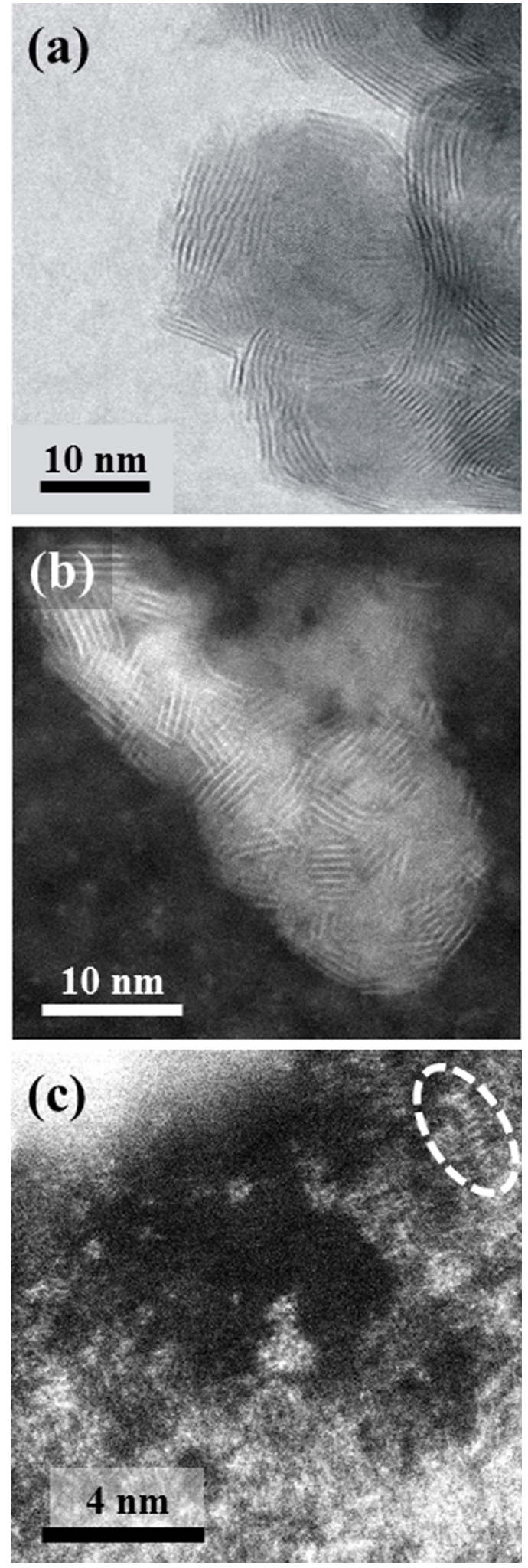

FIG. 5. High resolution bright field (a) and annular dark field (b) and (c) scanning transmission electron microscope images of $\mathrm{W}_{\mathrm{n}} \mathrm{S}_{\mathrm{m}}$ particles deposited on a silicon oxide TEM grid. The deposited material is agglomerated in larger lumps of material. The images show only bulk-like layered structures. At maximum resolution (c), individual metal atoms can be discerned (white dots). Stacks of very small platelets can be identified (white ellipse).

The cluster anions can be decelerated prior to deposition. This is achieved by an electric field between the substrate and the last element of the ion optics. If the voltage
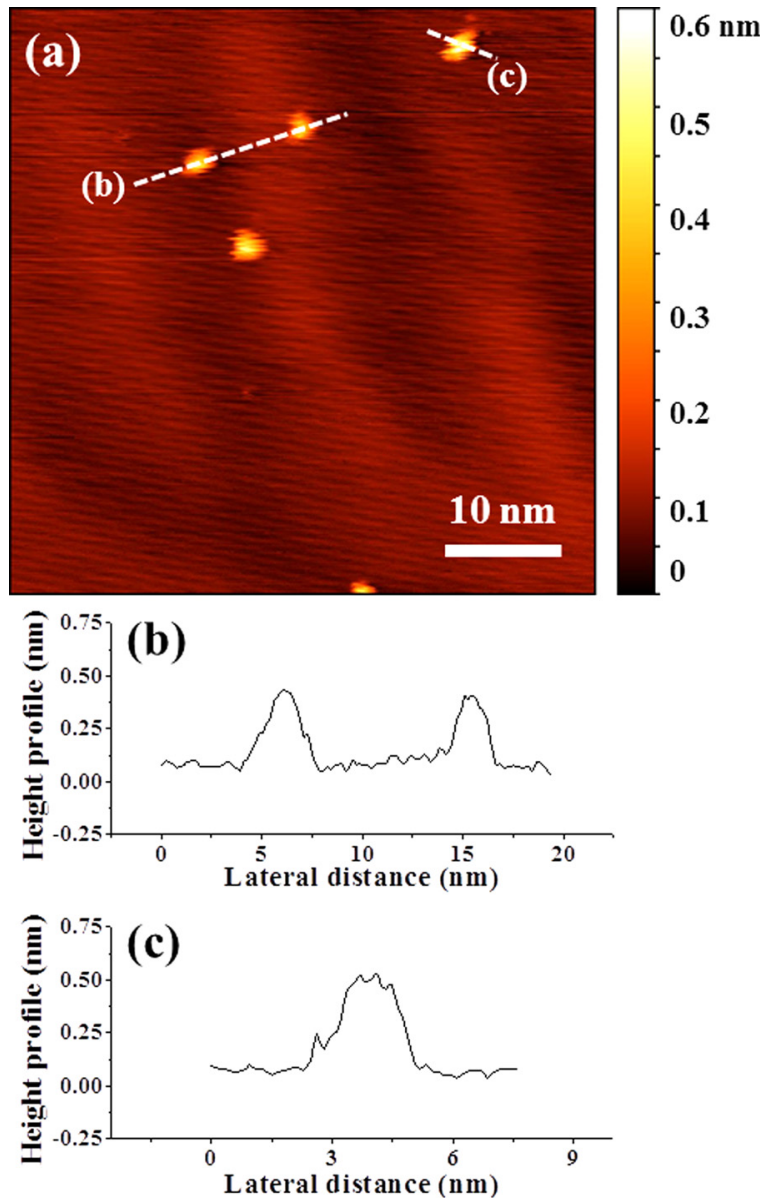

FIG. 6. Picture of a scanning tunneling microscope operated in ultrahigh vacuum of $\mathrm{Mo}_{\mathrm{n}} \mathrm{S}_{\mathrm{m}}{ }^{-}$cluster anions deposited at high kinetic energies (3-7 eV/atom). The clusters are pinned to defects on the terraces of the HOPG created by the impact. The height of the particles corresponds roughly to the thickness of a single layer of $\mathrm{MoS}_{2}$.

is set to $2.5 \mathrm{keV}$, practically no anions reach the surface. This is the consequence of the rather broad kinetic energy distribution covering the range between 1.5 and $2.5 \mathrm{keV}$. Therefore, a lower voltage is selected $(2000 \mathrm{~V})$ and now, clusters with not only very low energies but also with kinetic energies up to $500 \mathrm{eV}$ are deposited. We estimate the kinetic energies to vary between 0 and $3 \mathrm{eV} / \mathrm{atom}$. In general, soft landing conditions require a kinetic energy lower than $1 \mathrm{eV} /$ atom. But despite our rather undefined deposition conditions, now practically no pinned clusters are found on the terraces. Instead, most particles agglomerated at the step edges, indicating soft-landing conditions. Fig. 7 displays a high resolution STM image of $\mathrm{Mo}_{\mathrm{n}} \mathrm{S}_{\mathrm{m}}$ particles bound to a HOPG step edge. It is not possible to extract information about the internal structure of the particles from this picture, but it is possible to measure the height. The height scan provides a value of $0.38 \mathrm{~nm}$. The distance between the outer metal atoms in a single Mo-S-Mo layer is $0.32 \mathrm{~nm}$. Hence, the measured height of the deposited clusters is close to the thickness of a platelet. No cluster with a height significantly exceeding this value has been found in our deposition experiments.

None of our experimental results support the hypothesis of the existence of three-dimensional fullerene-like $\mathrm{Mo}_{\mathrm{n}} \mathrm{S}_{\mathrm{m}}$ 

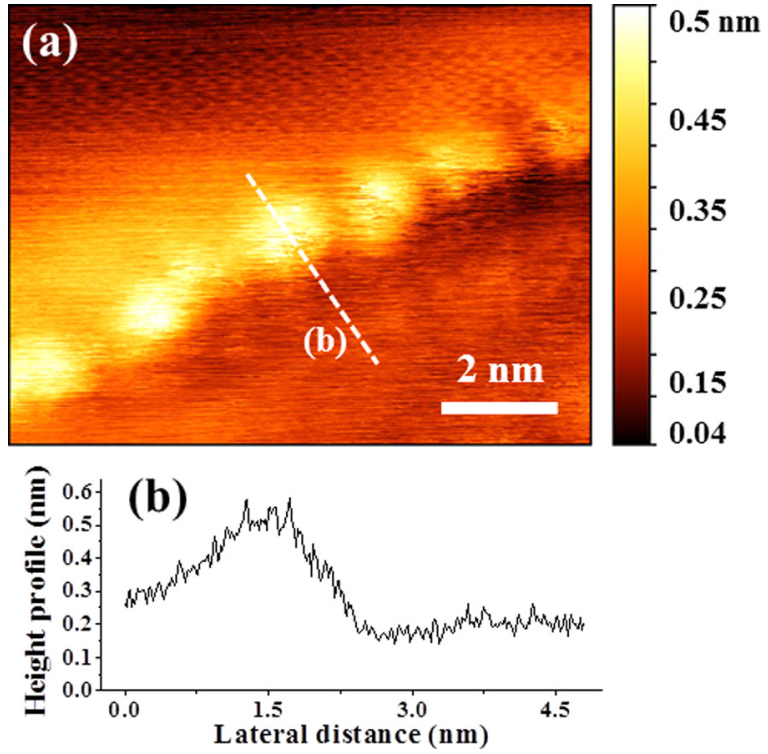

FIG. 7. STM picture of $\mathrm{Mo}_{\mathrm{n}} \mathrm{S}_{\mathrm{m}}{ }^{-}$clusters deposited on HOPG at lower kinetic energy ( $0-3 \mathrm{eV} / \mathrm{atom})$. The clusters diffuse to step edges. The height scans indicate a height corresponding to the thickness of a $\mathrm{MoS}_{2}$ platelet.

and $\mathrm{W}_{\mathrm{n}} \mathrm{S}_{\mathrm{m}}$ clusters in the second maximum of the mass spectrum of the annealed cluster anions. In contrast, the measurements are in full agreement with a two-dimensional platelet structure. It is still possible that some species in the gas phase do have a three-dimensional spherical or octahedral structure. But if this is the case, they are so fragile that they are destroyed by the deposition process. However, if the three-dimensional structure is so fragile, how can it be formed in the rather violent annealing discharge? It seems more likely that the clusters in the second maximum are just bulk-like clusters consisting of either larger platelets or stacks of smaller platelets as indicated by some of the STEM results (Fig. 5, white ellipse).

One question remains: Which process is responsible for the appearance of the second maximum? Without the annealing, the mass spectrum might extend to very large clusters, which are difficult to be detected by the time-offlight mass spectrometer. Most likely, the annealing discharge causes strong fragmentation and the large clusters shrink. Therefore, fragmentation of large clusters might give rise to the appearance of the second maximum. This would explain why it is possible to shift the second maximum back and forth by small changes of the source conditions (Fig. 3). If this explanation is correct, the bimodal structure would not be related to a change in the geometric structure. Instead, it would be the result of dynamical growth and fragmentation processes in the source.

We conclude that the clusters in the second maximum of the annealed time-of-flight mass spectrum most likely are two-dimensional platelets or stacks of platelets. Apparently, there are no fullerene-like single-wall metal chalcogenide clusters. The driving force for a graphene flake to bend itself into a nanosphere is the dangling bond at the edges of the flake. In the case of the sulfides, surplus sulfur atoms may saturate these dangling bonds. As long as there is the tiniest amount of excess sulfur, stabilization of a two-dimensional platelet structure might be energetically favorable to the curvature of the platelet. In our experiment, it is not possible to control the amount of sulfur so carefully that there is no excess sulfur. A reduction of the sulfur concentration below the stoichiometric required amount results in the formation of metal rich clusters, which have a three-dimensional compact structure. ${ }^{23}$ The system always has the opportunity to respond to a lack of sulfur by the formation of such metalrich species. Therefore, it might be impossible to force the growth of fullerene-like particles, because there will be always sulfur to stabilize the edges. The other possible hollow structure is an octahedron build from eight triangular platelets. Since the platelets are stable on their own, the bonds between the platelets may be weak. Therefore, singlewall octahedrons might be quite fragile and stacks of platelets may be more stable. Our findings are in agreement with an earlier experimental and theoretical study on small fullerene-like $\mathrm{MoS}_{2}$ clusters. The calculations predicted that single-wall species might be unstable. ${ }^{17}$

\section{CONCLUSION}

The driving force for the formation of carbon fullerenes are the dangling bonds at the edges of graphene flakes. To minimize the edge, the flake bends itself into a sphere. A similar mechanism might exist for all layered materials, such as $\mathrm{MoS}_{2}$ and $\mathrm{WS}_{2}$. In an effort to synthesize so-called "inorganic fullerenes," we generated small and medium-size $\mathrm{Mo}_{\mathrm{n}} \mathrm{S}_{\mathrm{m}}$ and $\mathrm{W}_{\mathrm{n}} \mathrm{S}_{\mathrm{m}}$ clusters with a cluster source optimized for the production of small carbon fullerenes. Similar to carbon, the very small $\mathrm{Mo}_{\mathrm{n}} \mathrm{S}_{\mathrm{m}}$ and $\mathrm{W}_{\mathrm{n}} \mathrm{S}_{\mathrm{m}}$ clusters consisting of less than 30 metal atoms are two-dimensional. Carbon clusters in this size regime are rings, while the metal chalcogenide clusters are platelets. The structure determination of the larger $\mathrm{Mo}_{\mathrm{n}} \mathrm{S}_{\mathrm{m}}$ and $\mathrm{W}_{\mathrm{n}} \mathrm{S}_{\mathrm{m}}$ clusters with more than 60 metal atoms turned out to be difficult. These clusters have been studied with mass spectroscopy, photoelectron spectroscopy, scanning transmission electron microscopy, and scanning tunneling microscopy. Disappointingly, all data indicate a two-dimensional bulk-like structure of these species similar to the structure of the very small clusters. There are no magic numbers, and the mass and photoelectron spectra are featureless. This difference to carbon might be explained by the additional degree of freedom a two-element system has. The edges of $\mathrm{MoS}_{2}$ and $\mathrm{WS}_{2}$ flakes can be stabilized by additional sulfur atoms. It is almost impossible to avoid this stabilization in a two-element condensation process. Hence, it could be that carbon and boron are the only materials able to form real fullerenes.

\section{ACKNOWLEDGMENTS}

Support by German Science Foundation (Grant No. GA 389/15-1) is gratefully acknowledged. S.J.P. acknowledges support from the U.S. Department of Energy, Materials Science and Engineering Division, and J.C.I. acknowledges support from ORNL's Center for Nanophase Materials Sciences, which is a U.S. Department of Energy, Office of Science User Facility. 
${ }^{1}$ M. S. Dresselhaus, G. Dresselhaus, and P. C. Eklund, The Science of Fullerenes and Carbon Nanotubes (Academic Press, Inc., 1996).

${ }^{2}$ A. A. Popov, S. Yang, and L. Dunsch, Chem. Rev. 113, 5989 (2013).

${ }^{3}$ P. Miró, M. Audiffred, and T. Heine, Chem. Soc. Rev. 43, 6537 (2014).

${ }^{4}$ H.-J. Zhai, Y.-F. Zhao, W.-L. Li, Q. Chen, H. Bai, H.-S. Hu, Z. A. Piazza, W.-J. Tian, H.-G. Lu, Y.-B. Wu, Y.-W. Mu, G.-F. Wei, Z.-P. Liu, J. Li, S.D. Li, and L.-S. Wang, Nat. Chem. 6, 727 (2014).

${ }^{5}$ Q. Chen, W.-L. Li, Y.-F. Zhao, S.-Y. Zhang, H.-S. Hu, H. Bai, H.-R. Li, W.-J. Tian, H.-G. Lu, H.-J. Zhai, S.-D. Li, J. Li, and L.-S. Wang, ACS Nano 9, 754 (2015).

${ }^{6}$ N. G. Chopra, R. J. Luyken, K. Cherrey, V. H. Crespi, M. L. Cohen, S. G. Louie, and A. Zettl, Science 269, 966 (1995).

${ }^{7}$ M. Remskar, A. Mrzel, Z. Skraba, A. Jesih, M. Ceh, J. Demsar, P. Stadelmann, F. Levy, and D. Mihailovic, Science 292, 479 (2001).

${ }^{8}$ R. Tenne, Colloids Surf., A 208, 83 (2002).

${ }^{9}$ Y. Q. Zhu and H. W. Kroto, J. Am. Chem. Soc. 125, 1329 (2003).

${ }^{10}$ M.-B. Sadan, L. Houben, A. N. Enyashin, G. Seifert, and R. Tenne, Proc. Natl. Acad. Sci. U.S.A. 105, 15643 (2008).

${ }^{11}$ R. Tenne, M. Resmkar, A. Enyashin, and G. Seifert, "Inorganic Nanotubes and Fullerene-Like Structures (IF)" in Carbon Nanotubes, edited by A. Jorio, G. Dresselhaus, and M. S. Dresselhaus (Springer, 2008), Vol. 111, pp. 631-671.

${ }^{12}$ R. Tenne and G. Seifert, Annu. Rev. Mater. Res. 39, 387 (2009).

${ }^{13}$ A. Yella, M. Panthöfer, M. Kappl, and W. Tremel, Angew. Chem. Int. Ed. 49, 2575 (2010).

${ }^{14}$ G. Radovsky, R. Popovitz-Biro, M. Staiger, K. Gartsman, C. Thomsen, T. Lorenz, G. Seifert, and R. Tenne, Angew. Chem. Int. Ed. 50, 12316 (2011).

${ }^{15}$ L. Yadgarov, R. Rosentsveig, G. Leitus, A. Albu-Yaron, A. Moshkovich, V. Perfilyev, R. Vasic, A. I. Frenkel, A. N. Enyashin, G. Seifert, L. Rapoport, and R. Tenne, Angew. Chem. Int. Ed. 51, 1148 (2012).

${ }^{16}$ R. Tenne, Front. Phys. 9, 370 (2014).
${ }^{17}$ M. Bar-Sadan, A. N. Enyashin, S. Gemming, R. Popovitz-Biro, S. Y. Hong, Y. Prior, R. Tenne, and G. Seifert, J. Phys. Chem. B 110, 25399 (2006).

${ }^{18}$ M. Nath, A. Govindaraj, and C. N. R. Rao, Adv. Mater. 13, 283 (2001).

${ }^{19}$ I. Wiesel, H. Arbel, R. Popovitz-Biro, J. M. Gordon, D. Feuermann, and R. Tenne, Nano Res. 2, 416 (2009).

${ }^{20}$ P. A. Parilla, A. C. Dillon, K. M. Jones, G. Riker, D. L. Schulz, D. S. Ginley, and M. J. Heben, Nature 397, 114 (1999).

${ }^{21}$ M. V. Bollinger, J. V. Lauritsen, K. W. Jacobsen, J. K. Norskov, S. Helveg, and F. Besenbacher, Phys. Rev. Lett. 87, 196803 (2001).

${ }^{22}$ J. V. Lauritsen, J. Kibsgaard, S. Helveg, H. Topsoe, B. S. Clausen, E. Laegsgaard, and F. Besenbacher, Nat. Nanotechnol. 2, 53 (2007).

${ }^{23}$ N. Bertram, Y. D. Kim, G. Ganteför, Q. Sun, P. Jena, J. Tamuliene, and G. Seifert, Chem. Phys. Lett. 396, 341 (2004).

${ }^{24}$ N. Bertram, J. Cordes, Y. D. Kim, S. Gemming, G. Seifert, and G. Ganteför, Chem. Phys. Lett. 418, 36 (2006).

${ }^{25}$ S. Gemming, J. Tamuliene, G. Seifert, N. Bertram, Y. D. Kim, and G. Ganteför, Appl. Phys. A 82, 161 (2006).

${ }^{26}$ E. A. Rohlfing, D. M. Cox, and A. Kaldor, J. Chem. Phys. 81, 3322 (1984).

${ }^{27}$ H. Kroto, J. R. Heath, S. C. O'Brien, R. F. Curl, and R. E. Smalley, Nature 318, 162 (1985).

${ }^{28}$ Y. Feldman, E. Wassermann, D. J. Srolovitz, and R. Tenne, Science 267, 222 (1995).

${ }^{29}$ D. M. David Jeba Singh, T. Pradeep, J. Bhattacharjee, and U. V. Waghmare, J. Phys. Chem. A 109, 7339 (2005).

${ }^{30}$ H. Handschuh, G. Ganteför, B. Kessler, P. S. Bechthold, and W. Eberhardt, Phys. Rev. Lett. 74, 1095 (1995).

${ }^{31}$ O. Gunnarsson, H. Handschuh, P. S. Bechthold, B. Kessler, G. Ganteför, and W. Eberhardt, Phys. Rev. Lett. 74, 1875 (1995).

${ }^{32}$ G. Schulze Icking-Konert, H. Handschuh, P. S. Bechthold, G. Ganteför, B. Kessler, and W. Eberhardt, Surf. Rev. Lett. 3, 483 (1996).

${ }^{33}$ M. Willis, M. Götz, A. K. Kandalam, G. F. Ganteför, and P. Jena, Angew. Chem. Int. Ed. 49, 8966 (2010). 